\title{
In silico-based experiments on mechanistic interactions between several intestinal permeation enhancers with a lipid bilayer model.
}

\author{
Authors: Rosita Kneiszl, ${ }^{\dagger, \ddagger}$ Shakhawath Hossain ${ }^{\dagger, \ddagger, \S}$ and Per Larsson $*, \dagger, \ddagger$
}

†Department of Pharmacy, Uppsala University, Husargatan 3, 75123 Uppsala, Sweden

†SweDeliver, Uppsala University, Husargatan 3, 75123 Uppsala, Sweden

${ }^{\S}$ Equal contribution: analyses and writing

*Corresponding Author

Table of contents:

\begin{tabular}{l|c}
\hline List of Figures & Page number \\
\hline $\begin{array}{l}\text { Supplementary Figure 1: Variation of PE molecules with time that remains } \\
\text { incorporated into the membrane leaflet where they were initially inserted }\end{array}$ & 2 \\
$\begin{array}{l}\text { Supplementary Figure 2: Interactions of caprate, caprylate and SNAC (all with } \\
\text { negative charges) with POPC membrane using CG-MD simulations. Snapshot } \\
\text { of (a) the initial and (b) final system configurations of the system with 35\% } \\
\text { concentrations of PEs were added. }\end{array}$ & 3 \\
$\begin{array}{l}\text { Supplementary Figure 3: The average order parameter of different PE } \\
\text { molecules }\end{array}$ & 4 \\
$\begin{array}{l}\text { Supplementary Figure 4: Fractional interaction matrix of caprate (with neutral } \\
\text { headgroups) and Sucrose monolaurate with membrane POPC molecules at } \\
\text { different PE concentration }\end{array}$ & 5 \\
\end{tabular}

\section{List of Tables}

Supplementary Table 1. A detailed description of the simulated systems with vaious PE concentrations used for each PE. 

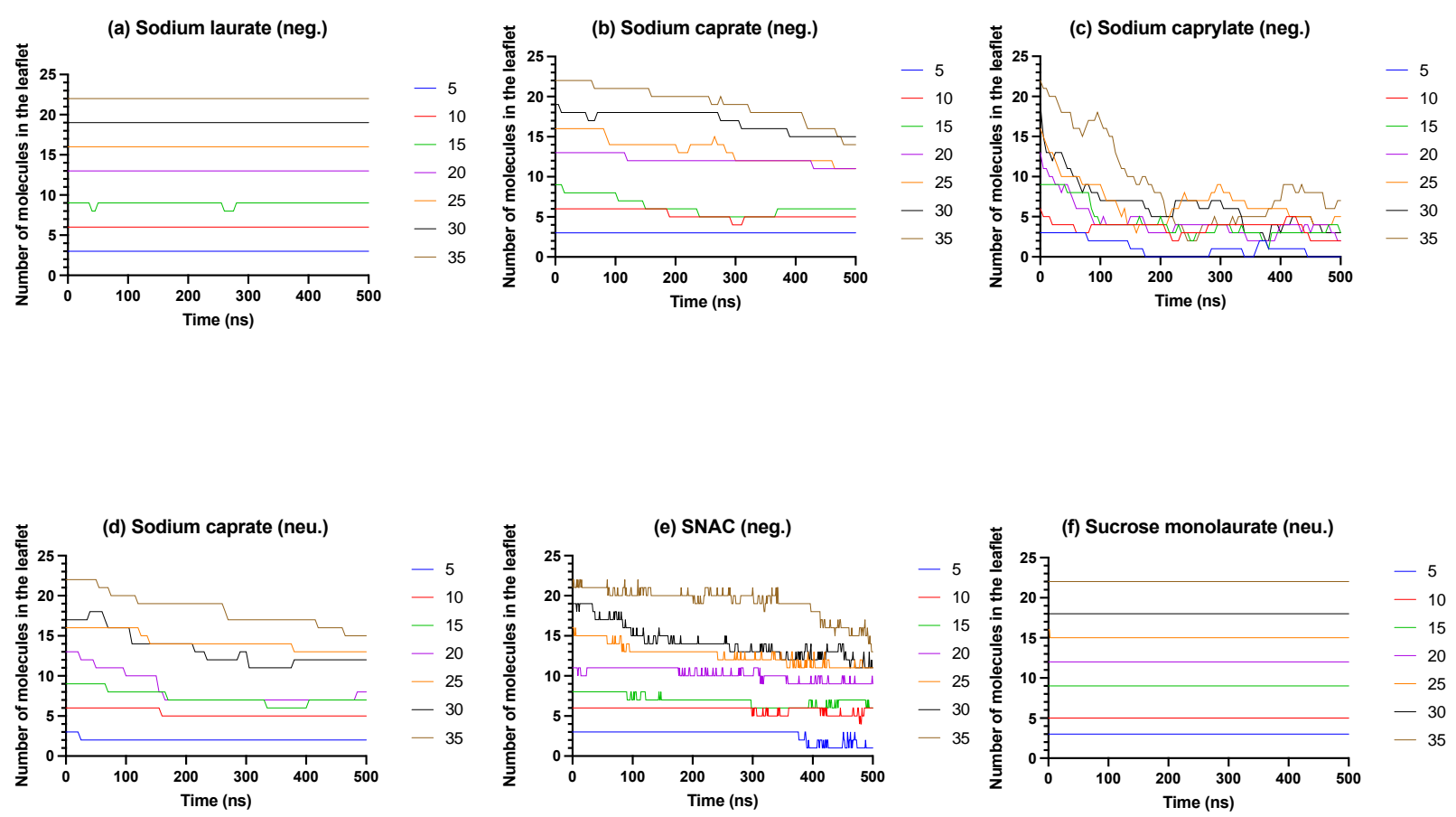

Supplementary Figure 1. The variation of the number of PE molecules over time that remains incorporated into the membrane leaflet in which they were initially inserted. All PE molecules except neutral sodium caprate did expel from the membrane. Neutral sodium caprate (d) did translocate within the membrane (flip-flop). Figures (a) neg. sodium laurate, (b) neg. sodium caprate, (c) neg. sodium caprylate, (d) neutral sodium caprate, note that all of the changes in the number of molecules is due to translocation within the membrane, so called flipflop events, from the "apical" side to the "basolateral" side of the model membrane, thus no expulsion events, (e) neg. SNAC (f) neutral sucrose monolaurate. 
(a) INITIAL SNAPSHOT Sodium caprate (neg.)

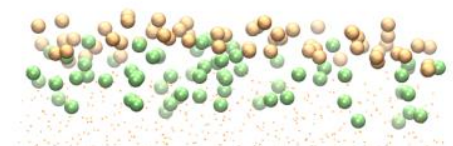

$\pi^{2} \cos ^{2}$

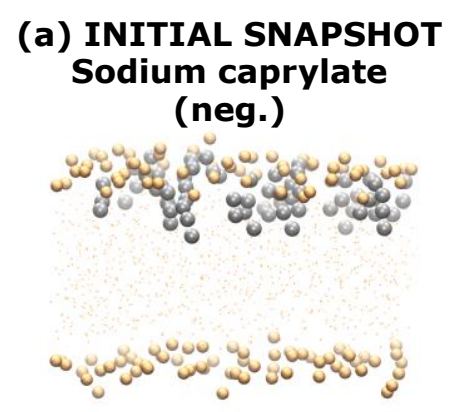

(a) INITIAL SNAPSHOT SNAC (neg.)

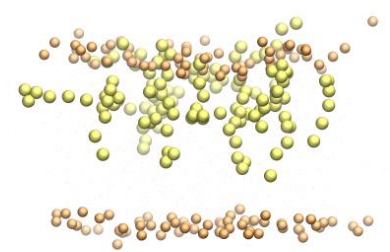

(b) FINAL SNAPSHOT Sodium caprate (neg.)

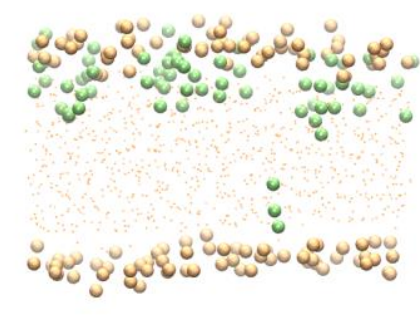

(b) FINAL SNAPSHOT Sodium caprylate (neg.)
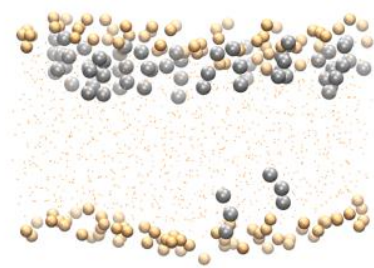

(b) FINAL SNAPSHOT SNAC (neg.)
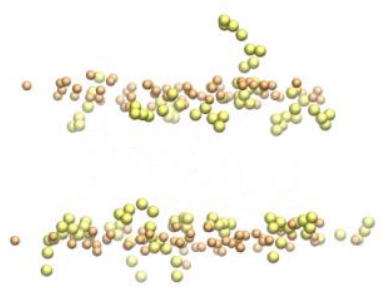

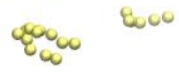

Supplementary Figure 2. Interactions of caprate, caprylate and SNAC (all with negative charges) with POPC membrane using CG-MD simulations. Snapshot of (a) the initial and (b) final system configurations of the system with $35 \%$ concentrations of PEs were added. 


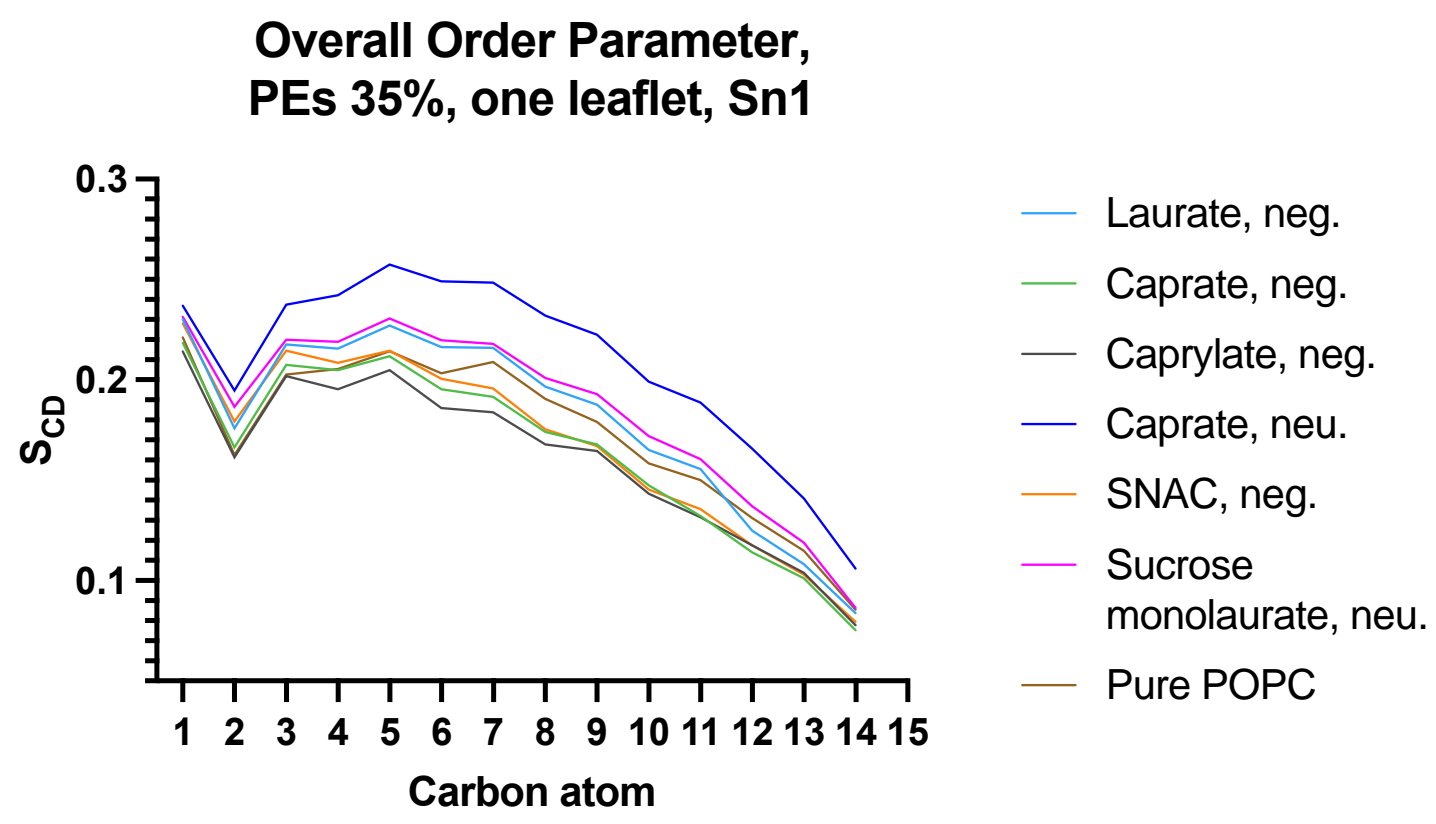

Supplementary Figure 3. The average order parameter of different PE molecules. 


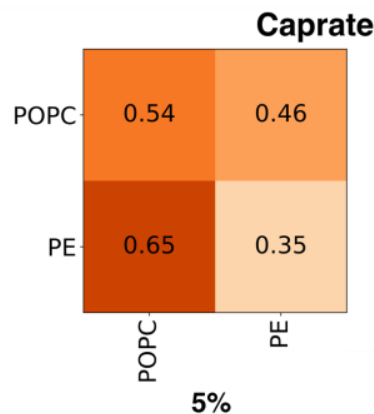

(neutral)

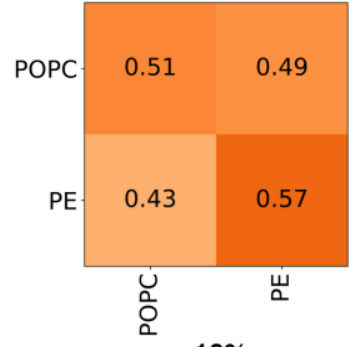

$10 \%$
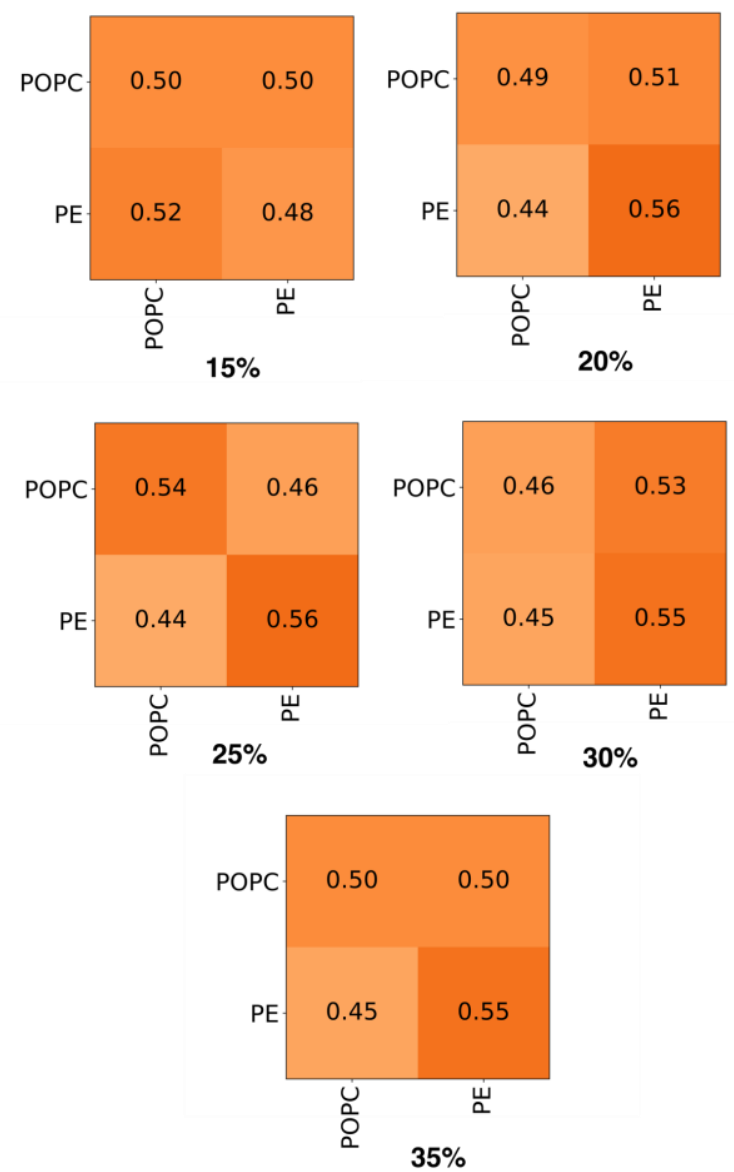

Sucrose monolaurate
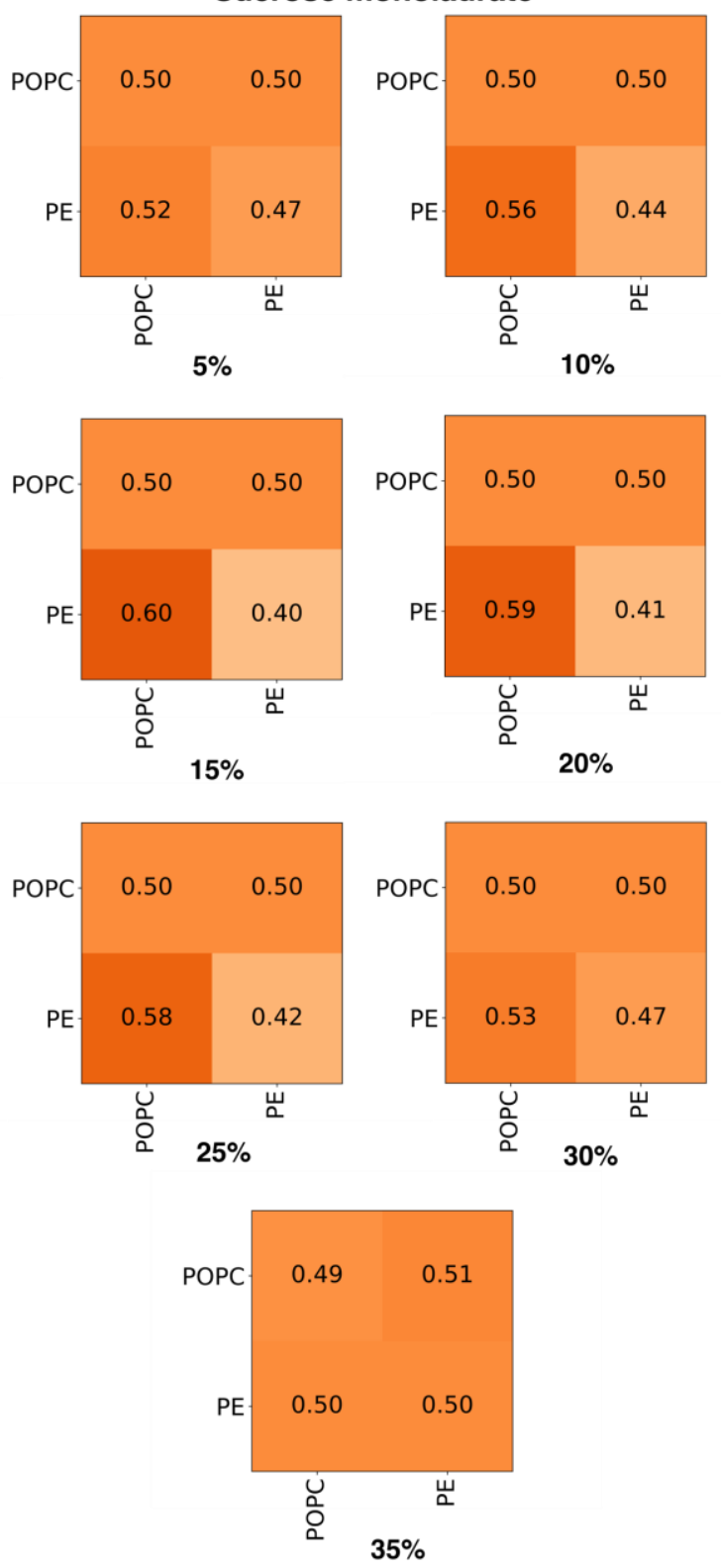

Supplementary Figure 4. Fractional interaction matrix of caprate (with neutral headgroups) and Sucrose monolaurate with membrane POPC molecules at different PE concentration. 
Supplementary Table 1. A detailed description of the simulated systems with vaious PE concentrations used for each PE.

\begin{tabular}{|c|c|c|c|c|c|c|c|}
\hline System $^{\text {I }}$ & $\mathrm{C}^{\mathrm{II}}(\%)$ & NumberIII & $\begin{array}{c}X^{I V} \\
(\mathbf{n m})\end{array}$ & $Y^{I V}(\mathrm{~nm})$ & $Z^{\mathrm{V}}(\mathrm{nm})$ & $\mathrm{C}(\mathbf{M})$ & $\mathrm{C}^{\mathrm{VI}}(\mathrm{mM})$ \\
\hline $\mathbf{0}$ & $\mathbf{0}$ & $\mathbf{0}$ & 6.45 & 6.45 & 4.93 & 0.00 & 0.00 \\
\hline 1 & 5 & 3 & 6.48 & 6.48 & 4.93 & 0.024 & 24.1 \\
\hline 2 & 10 & 6 & 6.52 & 6.52 & 4.93 & 0.048 & 47.7 \\
\hline 3 & 15 & 9 & 6.55 & 6.55 & 4.93 & 0.071 & 70.7 \\
\hline 4 & 20 & 13 & 6.60 & 6.60 & 4.93 & 0.100 & 100.5 \\
\hline 5 & 25 & 16 & 6.65 & 6.65 & 4.93 & 0.122 & 122 \\
\hline 6 & 30 & 19 & 6.67 & 6.67 & 4.93 & 0.144 & 144 \\
\hline 7 & 35 & 22 & 6.72 & 6.72 & 4.93 & 0.164 & 164 \\
\hline
\end{tabular}

ISystem 0 where no PEs were present was simulated only once. System 1 to 7 were replicated for each PE showed in Table 1.

IIPE number concentrations (\%)

IIIThe number of PE molecules inserted in one leaflet in each system.

${ }^{\text {IV }}$ The average lengths of the axes at that particular concentration for all six different types of PE molecules.

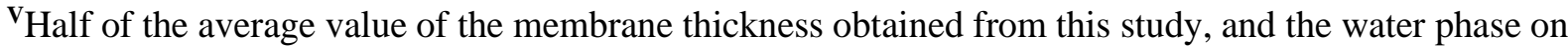
one side of the bilayer. NB: not the total value of the z-axis.

${ }^{\mathrm{VI}}$ The millimolar concentration. 